\title{
Direct Atomic-Scale Imaging of Multistep Phase Transition during the Lithiation of Nanowires by In-Situ (S)TEM
}

\author{
Anmin Nie, ${ }^{1,3}$ Robert F. Klie, ${ }^{3}$ Sreeram Vaddiraju, ${ }^{2}$ Reza Shahbazian -Yassar ${ }^{1,3,4}$ \\ ${ }^{1}$ Department of Mechanical Engineering-Engineering Mechanics, Michigan Technological \\ University, 1400 Townsend Dive, Houghton, Michigan 49931, USA \\ ${ }^{2}$ Artie McFerrin Department of Chemical Engineering, Texas A\&M University, \\ 3122 TAMU, College Station, TX 77843, USA \\ ${ }^{3}$ Department of Physics, University of Illinois at Chicago, Chicago, Illinois 60607, USA \\ ${ }^{4}$ Mechanical and Industrial Engineering Department, University of Illinois at Chicago, \\ Chicago, Illinois 60607, USA
}

$\mathrm{Sb}$-based alloy, such as $\mathrm{Zn}-\mathrm{Sb}^{1-3}$, are promising anode materials because of their high theoretical capacities and suitable operating voltages. However, there have been very few studies on the detailed dynamical process of the phase transition during lithiation in the Sb-based intermetallic compounds, especially at atomic scale. By employing in-situ (scanning) transmission electron microscopy ${ }^{4}$, we studied the lithium-ion diffusion and multistep phase transition during the electrochemical lithiation of individual single-crystal $\mathrm{Zn}_{4} \mathrm{Sb}_{3}$ nanowires with atomic-resolution. Continuous phase transition from crystalline rhombohedral $\mathrm{Zn}_{4} \mathrm{Sb}_{3}$ to hexagonal $\mathrm{LiZnSb}$ and then to cubic $\mathrm{Li}_{2} \mathrm{ZnSb}$ phases has been directly monitored upon successive lithium-ion intercalation, which is mediated by the formation of amorphous $\mathrm{Li}_{\mathrm{x}} \mathrm{Zn}_{4} \mathrm{Sb}_{3}$ at the early stage of lithiation. The kinetics of lithiation has been found to be highly anisotropic and relevant to the dynamics of the interfacial structures of the reaction front at different stages of lithiation.

\section{References:}

1.Park, C. M. \& Sohn, H. J. Adv. Mater. 22, (2010), 47.

2.Zhao, X. \& Cao, G. Electrochim. Acta 46, (2001), 891.

3.Xu, J., Wu, H., Wang, F., Xia, Y. \& Zheng, G. Adv. Energy Mater. 3, (2013), 286.

4.Nie A., \& Shahbazian -Yassar, R. ACSNano 7, (2013), 6203 


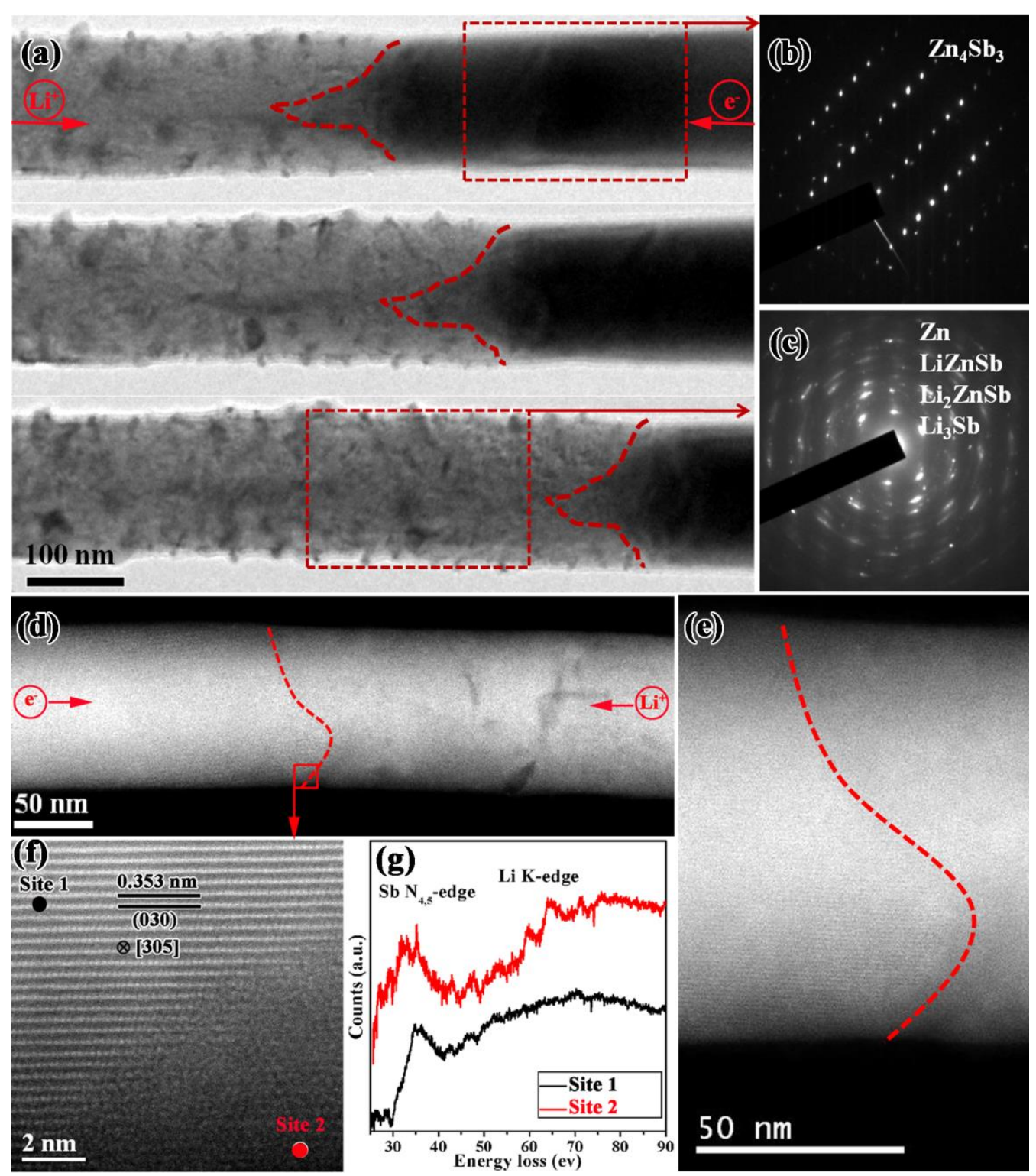

Figure 1 (a) TEM images recording the reaction front migration of the individual $\mathrm{Zn}_{4} \mathrm{Sb}_{3}$ nanowire during charging $-3.0 \mathrm{~V}$ against lithium metal coating with $\mathrm{Li}_{2} \mathrm{O}$ layer. (b) Electron diffraction pattern of the un-lithiated part of the nanowire indicated by the box, which is determined to be single crystal $\mathrm{Zn}_{4} \mathrm{Sb}_{3}$. (c) Electron diffraction pattern of the lithiated part of the nanowire, which shows that $\mathrm{Zn}$, $\mathrm{LiZnSb}, \mathrm{Li}_{2} \mathrm{ZnSb}$, and $\mathrm{Li3Sb}$ are formed after lithiation. (d) Low-mag HAADF image of a partial lithiated nanowire, which clear show a wedge interface between the lithiated and un-lithiated part in the nanowire. (e) High-mag HAADF image of the interface area. (f) Atomic scale resolution HAADF image of the interface, which indicates a lithiation-induced amorphous-crystalline interface at the early stage during charging. (g) EELS spectra taken from crystal and amorphous parts (Site 1 and Site $\mathrm{t} 2$ in panel $\mathrm{f}$ ) of the nanowire indicating the existence of the lithium element in the lithiated part. 